CARPATHIAN J. MATH.

Volume 37 (2021), No. 2,

Pages 217 - 226
Online version at https : //www . carpathian. cunbm . utcluj. ro/

Print Edition: ISSN 1584 - 2851; Online Edition: ISSN 1843 - 4401

DOI: https://doi.org/10.37193/CJM.2021.02.08

Dedicated to Prof. Ioan A. Rus on the occasion of his $85^{\text {th }}$ anniversary

\title{
Another characterization of hyperbolic diameter diminishing to zero IFSs
}

\author{
Radu Miculescu, Alexandru Mihail and CRistina-Maria PăCURAR
}

\begin{abstract}
In this paper we provide another characterization of hyperbolic diameter diminishing to zero iterated function systems that were studied in [R. Miculescu, A. Mihail, Diameter diminishing to zero IFSs, arXiv:2101.12705]. The primary tool that we use is an operator $H_{\mathcal{S}}$, associated to the iterated function system $\mathcal{S}$, which is inspired by the similar one utilized in Mihail (Fixed Point Theory Appl., 2015:75, 2015). Some fixed point results are also obtained as byproducts of our main result.
\end{abstract}

\section{INTRODUCTION}

This paper deals with iterated function systems that were introduced by J. Hutchinson (see [7]) and popularized by M. Barsnley (see [3]). As they induce a large and important class of fractal sets, a lot of generalizations of this concept have been studied. One of them is due to A. Kameyama (see [10]) who introduced a topological generalization of the attractor of an iterated function system. Let us mention some papers along this line of research: [1], [2], [4], [12], [13], [14], [15], [16], [17], [20] and [21]. From the point of view of this paper, an important role is played by [17] where the concept of (hyperbolic) diameter diminishing to zero iterated function system was introduced and its properties were studied. Moreover, using the concepts of hyperbolic $\varphi$-contractive iterated function system, hyperbolic (locally) uniformly point fibred iterated function system and iterated function system having hyperbolic attractor, characterizations of hyperbolic diameter diminishing to zero iterated function systems were provided.

Given an iterated function system $\mathcal{S}=\left((X, d),\left(f_{i}\right)_{i \in I}\right)$, we shall consider the following:

- $I^{\mathbb{N}^{*}} \stackrel{\text { not }}{=} \Lambda(I)$ endowed with the Baire metric which is denoted by $d_{\Lambda}$ (for details see Preliminaries)

$-\mathcal{C}(X)=\{f: \Lambda(I) \times X \rightarrow X \mid f$ is continuous and bounded $\}$ endowed with the uniform metric, $\Lambda(I) \times X$ being furnished with the metric $\rho$ given by $\rho((\omega, x),(\theta, y))=$ $\left\{\begin{array}{cc}d_{\Lambda}(\omega, \theta)+1, & \text { if } x \neq y \\ d_{\Lambda}(\omega, \theta), & \text { if } x=y\end{array}\right.$ for every $(\omega, x),(\theta, y) \in \Lambda(I) \times X$

- the operator $H_{\mathcal{S}}: \mathcal{C}(X) \rightarrow \mathcal{C}(X)$ acting as follows:

$$
H_{\mathcal{S}}(f)(\omega, x)=f_{\omega_{1}}\left(f\left(\omega_{2} \ldots \omega_{n} \ldots, x\right)\right),
$$

Received: 10.02.2021. In revised form: 24.03.2021. Accepted: 31.03.2021

2010 Mathematics Subject Classification. 28A80, 37C70, 54H20.

Key words and phrases. iterated function system (IFS), (hyperbolic) $\varphi$-contractive IFS, (hyperbolic) locally uniformly point fibred IFS, (hyperbolic) uniformly point fibred IFS, IFS having (hyperbolic) attractor, (hyperbolic) diameter diminishing to zero IFS, $H_{\mathcal{S}}$ operator, Picard operator.

Corresponding author: Miculescu Radu; radu.miculescu@unitbv.ro 
for every $f \in \mathcal{C}(X), \omega=\omega_{1} \omega_{2} \ldots \omega_{n} \ldots \in \Lambda(I)$ and $x \in X$. On the one hand, we prove that if $\mathcal{S}$ is $\varphi$-contractive, $\varphi$ being a comparison function, then $H_{\mathcal{S}}$ is a Picard operator (see Proposition 3.2). On the other hand, we prove that if $H_{\mathcal{S}}$ is a Picard operator, then $\mathcal{S}$ has attractor and admits canonical projection (see Theorem 3.3). In this way we provide another characterization of hyperbolic diameter diminishing to zero iterated function systems that were studied in [17].

\section{PRELIMINARIES}

By $\mathbb{N}$ we mean the set $\{0,1,2, \ldots, n, \ldots\}$ and by $\mathbb{N}^{*}$ we mean the set $\{1,2, \ldots, n, \ldots\}$.

By $B^{A}$, where $A$ and $B$ are two sets, we mean the set of functions from $A$ to $B$.

Given a function $f: X \rightarrow X$ and $n \in \mathbb{N}^{*}$, by $f^{[n]}$ we mean the composition of $f$ by itself $n$ times. By $f^{[0]}$ we mean the identity function $I d_{X}: X \rightarrow X$ given by $I_{X}(x)=x$ for every $x \in X$.

Given a metric space $(X, d)$, by:

- $P(X)$ we designate the class of all non-empty subsets of $X$

- $P_{b}(X)$ we designate the set of non-empty bounded subsets of $X$

- $P_{b, c l}(X)$ we designate the set of non-empty bounded and closed subsets of $X$

- $P_{c p}(X)$ we designate the set of non-empty compact subsets of $X$.

\section{The Hausdorff-Pompeiu metric.}

Definition 2.1. (Hausdorff-Pompeiu metric) Given a metric space $(X, d)$, the generalized Hausdorff-Pompeiu pseudometric is the function $\mathcal{H}_{d}: P(X) \times P(X) \rightarrow[0,+\infty]$ given by

$$
\mathcal{H}_{d}(A, B)=\max \left\{\sup _{x \in A} d(x, B), \sup _{x \in B} d(x, A)\right\}
$$

for all $A, B \in P(X)$.

The Hausdorff-Pompeiu metric is the function $H_{d}: P_{b, c l}(X) \times P_{b, c l}(X) \rightarrow[0,+\infty)$ given by

$$
H_{d}(A, B)=\mathcal{H}_{d}(A, B)
$$

for all $A, B \in P_{b, c l}(X)$.

Remark 2.1. In the framework of the above Definition, we have:

a) $\mathcal{H}_{d}(A, B)=\mathcal{H}_{d}(\bar{A}, \bar{B})$, for all $A, B \in P(X)$.

b) $\mathcal{H}_{d}\left(\cup_{i \in I} A_{i}, \cup_{i \in I} B_{i}\right) \leq \sup _{i \in I} \mathcal{H}_{d}\left(A_{i}, B_{i}\right)$, for any two families $\left(A_{i}\right)_{i \in I}$ and $\left(B_{i}\right)_{i \in I}$ of elements from $P(X)$.

\section{The shift space.}

Let $I$ be a non-empty set and $n \in \mathbb{N}^{*}$.

$\Lambda(I)$ is the set of infinite words with letters from the alphabet $I$ and a standard element $\omega$ of $\Lambda(I)$ can be presented as $\omega=\omega_{1} \omega_{2} \ldots \omega_{n} \omega_{n+1} \ldots$. So $\Lambda(I)$ is $I^{\mathbb{N}^{*}}$.

$\Lambda_{n}(I)$ is the set of words with letters from the alphabet $I$ of length $n$ and a standard element $\omega$ of $\Lambda_{n}(I)$ can be presented as $\omega=\omega_{1} \omega_{2} \ldots \omega_{n}$. So $\Lambda_{n}(I)$ is $I^{\{1,2, \ldots, n\}}$.

$\Lambda_{0}(I)$ is the set having only one element, namely the empty word denoted by $\lambda$.

Endowed with the distance described by

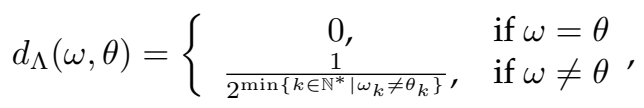

where $\omega=\omega_{1} \omega_{2} \omega_{3} \ldots \omega_{n} \omega_{n+1} \ldots$ and $\theta=\theta_{1} \theta_{2} \theta_{3} \ldots \theta_{n} \theta_{n+1} \ldots, \Lambda(I)$ becomes a metric space.

Note that $\left(\Lambda(I), d_{\Lambda}\right)$ is compact if $I$ is finite. 
For $m \in \mathbb{N}^{*}$ and $\omega=\omega_{1} \omega_{2} \ldots \omega_{n} \omega_{n+1} \ldots \in \Lambda(I)$, by $[\omega]_{m}$ we designate the word $\omega_{1} \omega_{2} \ldots \omega_{m} \in$ $\Lambda_{m}(I)$.

For $i \in I$, one can consider the function $\tau_{i}: \Lambda(I) \rightarrow \Lambda(I)$ given by

$$
\tau_{i}(\omega)=i \omega_{1} \omega_{2} \ldots \omega_{n} \omega_{n+1} \ldots,
$$

for every $\omega=\omega_{1} \omega_{2} \ldots \omega_{n} \omega_{n+1} \ldots \in \Lambda(I)$.

\section{Comparison functions and $\varphi$-contractions.}

Definition 2.2. (comparison function) A function $\varphi:[0, \infty) \rightarrow[0, \infty)$ is called a comparison function if: i) $\varphi$ is increasing; ii) $\varphi(t)<t$ for every $t>0$; iii) $\varphi$ is right-continuous.

Definition 2.3. ( $\varphi$-contraction) Given a metric space $(X, d)$ and a comparison function $\varphi$, a function $f: X \rightarrow X$ is called $\varphi$-contraction if

$$
d(f(x), f(y)) \leq \varphi(d(x, y)),
$$

for all $x, y \in X$.

\section{Picard operators.}

Definition 2.4. (Picard operator) Given a metric space $(X, d)$, a function $f: X \rightarrow X$ is called Picard operator if:

i) there exists a unique fixed point $\alpha$ of $f$;

ii)

$$
\lim _{n \rightarrow \infty} f^{[n]}(x)=\alpha
$$

for every $x \in X$.

Theorem 2.1. (see Theorem 1 from [6] or Theorem 1 from [8]) Given a complete metric space $(X, d)$ and a comparison function $\varphi$, each $\varphi$-contraction $f: X \rightarrow X$ is a Picard operator.

\section{Iterated function systems.}

Definition 2.5. (iterated function system) A pair $\left((X, d),\left(f_{i}\right)_{i \in I}\right)$ is called iterated function system (IFS for short) if:

i) $(X, d)$ is a complete metric space;

ii) $I$ is a finite set;

iii) $f_{i}: X \rightarrow X$ is continuous for each $i \in I$;

iv) $f_{i}(B) \in P_{b}(X)$ for every $B \in P_{b}(X)$ and every $i \in I$.

Under the framework of the above definition, in the sequel, we shall use the following notations:

- an IFS $\left((X, d),\left(f_{i}\right)_{i \in I}\right)$ will be denoted by $\mathcal{S}$;

- for $\omega=\omega_{1} \omega_{2} \ldots \omega_{n} \in \Lambda_{n}(I), f_{\omega_{1}} \circ \ldots \circ f_{\omega_{n}}$ will be denoted by $f_{\omega}$.

Definition 2.6. (fractal operator) Given an IFS $\mathcal{S}=\left((X, d),\left(f_{i}\right)_{i \in I}\right)$, the function

$$
F_{\mathcal{S}}: P_{b, c l}(X) \rightarrow P_{b, c l}(X),
$$

given by

$$
F_{\mathcal{S}}(B)=\overline{\bigcup_{i \in I} f_{i}(B)}
$$

for every $B \in P_{b, c l}(X)$, is called the fractal operator associated to $\mathcal{S}$.

Definition 2.7. ( $\varphi$-contractive IFS) Given a comparison function $\varphi$, an iterated function system $\mathcal{S}=\left((X, d),\left(f_{i}\right)_{i \in I}\right)$ is called $\varphi$-contractive if each $f_{i}$ is a $\varphi$-contraction.

Definition 2.8. (point fibred IFS, locally uniformly point fibred IFS, uniformly point fibred IFS) An iterated function system $\mathcal{S}=\left((X, d),\left(f_{i}\right)_{i \in I}\right)$ is called: 
a) point fibred if for every $\omega \in \Lambda(I)$ there exists $a_{\omega} \in X$ such that

$$
\lim _{n \rightarrow \infty} f_{[\omega]_{n}}(x)=a_{\omega}
$$

for all $x \in X$;

b) locally uniformly point fibred if it is point fibred and for each $x \in X$ there exists an open set $D_{x}$ containing $x$ such that

$$
\lim _{n \rightarrow \infty} \sup _{\omega \in \Lambda(I)} \sup _{y \in D_{x}} d\left(f_{[\omega]_{n}}(y), a_{\omega}\right)=0
$$

c) uniformly point fibred if it is point fibred and

$$
\lim _{n \rightarrow \infty} \sup _{\omega \in \Lambda(I)} \sup _{x \in B} d\left(f_{[\omega]_{n}}(x), a_{\omega}\right)=0,
$$

for every $B \in P_{b, c l}(X)$.

Definition 2.9. (diameter diminishing to zero iterated function system) An iterated function system $\mathcal{S}=\left((X, d),\left(f_{i}\right)_{i \in I}\right)$ is called diameter diminishing to zero iterated function systems if for every $B \in P_{b, c l}(X)$ there exists $M_{B} \in P_{b, c l}(X)$ such that:
i) $B \subseteq M_{B}$
ii) $F_{\mathcal{S}}\left(M_{B}\right) \subseteq M_{B}$
iii) $\lim _{n \rightarrow \infty} \max _{\omega \in \Lambda_{n}(I)} \operatorname{diam}\left(f_{\omega}\left(M_{B}\right)\right)=0$.

Definition 2.10. (IFS having attractor) We say that the iterated function system $\mathcal{S}=$ $\left((X, d),\left(f_{i}\right)_{i \in I}\right)$ has attractor if there exists $A_{\mathcal{S}} \in P_{b, c l}(X)$ such that:

i) $F_{\mathcal{S}}\left(A_{\mathcal{S}}\right)=A_{\mathcal{S}}$

ii) $\lim _{n \rightarrow \infty} H_{d}\left(F_{\mathcal{S}}^{[n]}(B), A_{\mathcal{S}}\right)=0$,

for each $B \in P_{b, c l}(X)$.

Remark 2.2. a) (see [5]) If $K \in P_{c p}(X)$ and $\left(K_{n}\right)_{n \in \mathbb{N}} \subseteq P_{c p}(X)$ are such that $\lim _{n \rightarrow \infty} H_{d}\left(K_{n}, K\right)=$ 0 , then $\lim _{n \rightarrow \infty} H_{d}\left(F_{\mathcal{S}}\left(K_{n}\right), F_{\mathcal{S}}(K)\right)=0$.

called the attractor of $\mathcal{S}$.

b) Actually $A_{\mathcal{S}}$ is the only fixed point of $F_{\mathcal{S}}$. It belongs to $P_{c p}(X)$ and it is

Definition 2.11. (hyperbolic $\varphi$-contractive IFS, hyperbolic locally uniformly point fibred IFS, hyperbolic uniformly point fibred IFS, IFS having hyperbolic attractor and hyperbolic diameter diminishing to zero IFS) Given a comparison function $\varphi$, an iterated function system $\mathcal{S}=\left((X, d),\left(f_{i}\right)_{i \in I}\right)$ is called hyperbolic $\varphi$-contractive if there exists a distance $d_{1}$ on $X$ such that:

i) $d$ and $d_{1}$ are topologically equivalent;

ii) $\left(X, d_{1}\right)$ is complete;

iii) $\mathcal{S}_{1}=\left(\left(X, d_{1}\right),\left(f_{i}\right)_{i \in I}\right)$ is $\varphi$-contractive.

The concepts of hyperbolic locally uniformly point fibred IFS, hyperbolic uniformly point fibred IFS, IFS having hyperbolic attractor and hyperbolic diameter diminishing to zero IFS are defined in a similar manner.

Let us recall the main result from [17].

Theorem 2.2. For an iterated function system $\mathcal{S}=\left((X, d),\left(f_{i}\right)_{i \in I}\right)$, the following statements are equivalent:

1. There exists a comparison function $\varphi$ such that $\mathcal{S}$ is hyperbolic $\varphi$-contractive.

2. $\mathcal{S}$ is hyperbolic locally uniformly point fibred.

3. $\mathcal{S}$ is hyperbolic uniformly point fibred.

4. $\mathcal{S}$ is a hyperbolic diameter diminishing to zero iterated function system.

5. $\mathcal{S}$ has hyperbolic attractor and there exists a continuous surjection $\pi: \Lambda(I) \rightarrow A_{\mathcal{S}_{1}}$ such that $\pi \circ \tau_{i}=f_{i} \circ \pi$ for all $i \in I$, where $\mathcal{S}_{1}$ is the IFS mentioned in Definition 2.11, iii). 


\section{THE MAIN RESULTS}

The metric space $\mathcal{C}(X)$.

Definition 3.12. (the metric space $\mathcal{C}(X))$ Given an IFS $\mathcal{S}=\left((X, d),\left(f_{i}\right)_{i \in I}\right)$, one can consider the metric space $\left(\mathcal{C}(X), d_{u}\right)$, where

$$
\begin{gathered}
\mathcal{C}(X)=\{f: \Lambda(I) \times X \rightarrow X \mid f \text { is continuous and bounded }\}, \\
d_{u}(f, g)=\sup _{\omega \in \Lambda(I), x \in X} d(f(\omega, x), g(\omega, x)),
\end{gathered}
$$

for every $f, g \in \mathcal{C}(X)$ and the metric $\rho$ on $\Lambda(I) \times X$ is given by

$$
\rho((\omega, x),(\theta, y))=\left\{\begin{array}{cc}
d_{\Lambda}(\omega, \theta)+1, & \text { if } x \neq y \\
d_{\Lambda}(\omega, \theta), & \text { if } x=y
\end{array}\right.
$$

for every $(\omega, x),(\theta, y) \in \Lambda(I) \times X$.

Remark 3.3. The metric space $\mathcal{C}(X)$ is complete.

The operator $H_{\mathcal{S}}$.

Definition 3.13. (the operator $\left.H_{\mathcal{S}}\right)$ Given an IFS $\mathcal{S}=\left((X, d),\left(f_{i}\right)_{i \in I}\right)$, one can consider the operator $H_{\mathcal{S}}: \mathcal{C}(X) \rightarrow \mathcal{C}(X)$ given by

$$
H_{\mathcal{S}}(f)(\omega, x)=f_{\omega_{1}}\left(f\left(\omega_{2} \ldots \omega_{n} \ldots, x\right)\right),
$$

for every $f \in \mathcal{C}(X), \omega=\omega_{1} \omega_{2} \ldots \omega_{n} \ldots \in \Lambda(I), x \in X$.

Note that the above defined operator $H_{\mathcal{S}}$ is inspired by the one introduced by A. Mihail in [18] but it maps $\mathcal{C}(X)$ into itself while the one used in [18] maps $\{f: \Lambda(I) \rightarrow X \mid$ f continuous $\}$ into itself.

Remark 3.4. $H_{\mathcal{S}}$ is well defined i.e. $H_{\mathcal{S}}(f) \in \mathcal{C}(X)$ for every $f \in \mathcal{C}(X)$.

Indeed, for every $f \in \mathcal{C}(X)$ and every $i \in I$, the restriction of $H_{\mathcal{S}}(f)$ to $\tau_{i}(\Lambda(I)) \times X$ is continuous as the functions $f_{i}, f$ and $\omega=\omega_{1} \omega_{2} \ldots \omega_{n} \ldots \in \Lambda(I) \rightarrow \omega_{2} \ldots \omega_{n} \ldots \in \Lambda(I)$ are continuous. Since $\Lambda(I) \times X=\cup_{i \in I}\left(\tau_{i}(\Lambda(I)) \times X\right)$ and $\tau_{i}(\Lambda(I)) \times X$ is an open subset of $\Lambda(I)$ for every $i \in I$, in view of Theorem 18.2 from [19], we conclude that $H_{\mathcal{S}}(f)$ is continuous. The fact that $H_{\mathcal{S}}(f)$ is bounded is a consequence of Definition 2.5, iv).

\section{Remark 3.5.}

$$
H_{\mathcal{S}}^{[n]}(f)(\omega, x)=f_{[\omega]_{n}}\left(f\left(\omega_{n+1} \omega_{n+2} \ldots \omega_{m} \ldots, x\right)\right),
$$

for every $n \in \mathbb{N}^{*}, \omega=\omega_{1} \omega_{2} \ldots \omega_{n} \ldots \in \Lambda(I), f \in \mathcal{C}(X)$ and $x \in X$.

Indeed, the above equality could be proved by induction on $n$.

One could see that $(*)$ is valid for $n=1$ just by the definition of the operator $H_{\mathcal{S}}$.

Let us suppose that $(*)$ is true for $n$.

Then

$$
\begin{gathered}
H_{\mathcal{S}}^{[n+1]}(f)(\omega, x)=H_{\mathcal{S}}^{[n]}\left(H_{\mathcal{S}}(f)\right)(\omega, x) \stackrel{(*) \text { is true for } n}{=} f_{[\omega]_{n}}\left(H_{\mathcal{S}}(f)\left(\omega_{n+1} \omega_{n+2} \ldots \omega_{m} \ldots, x\right)\right)= \\
=f_{[\omega]_{n}}\left(f_{\omega_{n+1}}\left(f\left(\omega_{n+2} \ldots \omega_{m} \ldots, x\right)\right)\right)=f_{[\omega]_{n+1}}\left(f\left(\omega_{n+2} \ldots \omega_{m} \ldots, x\right)\right),
\end{gathered}
$$

for every $\omega=\omega_{1} \omega_{2} \ldots \omega_{n} \ldots \in \Lambda(I)$ and $x \in X$, i.e. $(*)$ is true for $n+1$. 
The properties of $H_{\mathcal{S}}$.

Proposition 3.1. If there exists a comparison function $\varphi$ such that the iterated function system $\mathcal{S}=\left((X, d),\left(f_{i}\right)_{i \in I}\right)$ is $\varphi$-contractive, then $H_{\mathcal{S}}$ is a $\varphi$-contraction.

Proof. We have

$$
\begin{gathered}
d\left(H_{\mathcal{S}}(f)(\omega, x), H_{\mathcal{S}}(g)(\omega, x)\right)= \\
=d\left(f_{\omega_{1}}\left(f\left(\omega_{2} \ldots \omega_{n} \ldots, x\right)\right), f_{\omega_{1}}\left(g\left(\omega_{2} \ldots \omega_{n} \ldots, x\right)\right)\right) \stackrel{f_{\omega_{1}} \text { is } \varphi \text {-contraction }}{\leq} \\
\leq \varphi\left(d\left(f\left(\omega_{2} \ldots \omega_{n} \ldots, x\right), g\left(\omega_{2} \ldots \omega_{n} \ldots, x\right)\right)\right) \stackrel{\text { Definition 2.2, i) }}{\leq} \varphi\left(d_{u}(f, g)\right),
\end{gathered}
$$

for every $\omega=\omega_{1} \omega_{2} \ldots \omega_{n} \ldots \in \Lambda(I), x \in X, f, g \in \mathcal{C}(X)$, so

$$
d_{u}\left(H_{\mathcal{S}}(f), H_{\mathcal{S}}(g)\right)=\sup _{\omega \in \Lambda(I), x \in X} d\left(H_{\mathcal{S}}(f)(\omega, x), H_{\mathcal{S}}(g)(\omega, x)\right) \leq \varphi\left(d_{u}(f, g)\right),
$$

for every $f, g \in \mathcal{C}(X)$.

Proposition 3.2. If there exists a comparison function $\varphi$ such that the iterated function system $\mathcal{S}$ is $\varphi$-contractive, then $H_{\mathcal{S}}$ is a Picard operator.

Proof. We have just to use Theorem 2.1, Remark 3.3 and Proposition 3.1.

\section{Some properties of $\mathcal{S}$ in case that $H_{\mathcal{S}}$ is a Picard operator.}

Theorem 3.3. If the iterated function system $\mathcal{S}=\left((X, d),\left(f_{i}\right)_{i \in I}\right)$ has the property that $H_{\mathcal{S}}$ is a Picard operator and $p \in \mathcal{C}(X)$ is its fixed point, then:

i) for every $\omega \in \Lambda(I)$ there exists $a_{\omega} \in X$ such that

$$
p(\{\omega\} \times X)=\left\{a_{\omega}\right\} ;
$$

ii) $\mathcal{S}$ is point fibred;

iii) $\mathcal{S}$ has attractor;

iv) the function $\pi: \Lambda(I) \rightarrow A_{\mathcal{S}}$, given by

$$
\pi(\omega)=a_{\omega},
$$

for every $\omega \in \Lambda(I)$, where $A_{\mathcal{S}}$ is the attractor of $\mathcal{S}$, has the following properties:

a) it is continuous and onto;

b)

for all $i \in I$.

$$
\pi \circ \tau_{i}=f_{i} \circ \pi
$$

Proof. According to the hypothesis, we have

$$
H_{\mathcal{S}}(p)=p \quad \text { and } \quad \lim _{n \rightarrow \infty} H_{\mathcal{S}}^{[n]}(g)=p,
$$

for every $g \in \mathcal{C}(X)$.

i) For $u_{0} \in X$ arbitrarily chosen, but fixed, let us consider $g_{0} \in \mathcal{C}(X)$ given by

$$
g_{0}(\omega, x)=u_{0},
$$

for every $(\omega, x) \in \Lambda(I) \times X$.

Then

$$
\lim _{n \rightarrow \infty} H_{\mathcal{S}}^{[n]}\left(g_{0}\right)=p
$$


so

$$
\lim _{n \rightarrow \infty} H_{\mathcal{S}}^{[n]}\left(g_{0}\right)(\omega, x)=p(\omega, x),
$$

for every $x \in X$ and $\omega \in \Lambda(I)$.

Then, via Remark 3.5, we obtain

$$
\lim _{n \rightarrow \infty} f_{[\omega]_{n}}\left(g_{0}\left(\omega_{n+1} \omega_{n+2} \ldots \omega_{m} \ldots, x\right)\right)=p(\omega, x),
$$

i.e.

$$
\lim _{n \rightarrow \infty} f_{[\omega]_{n}}\left(u_{0}\right)=p(\omega, x),
$$

for every $x \in X$ and $\omega \in \Lambda(I)$.

Hence, for every $\omega \in \Lambda(I)$, the set $\{p(\omega, x) \mid x \in X\}$ is a singleton and the justification of i) is completed.

ii) It results from (3.1) which can be rewritten as

$$
\lim _{n \rightarrow \infty} f_{[\omega]_{n}}(x)=a_{\omega}
$$

for all $x \in X$.

iii) Let us choose $x_{0} \in X$ and note that

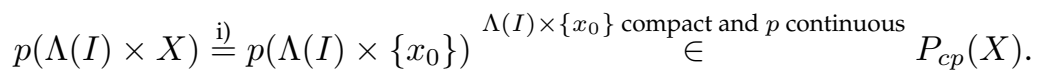

In the sequel, we shall use the following notation

$$
p(\Lambda(I) \times X) \stackrel{\text { not }}{=} A_{\mathcal{S}}
$$

\section{Claim 1}

$$
\lim _{n \rightarrow \infty} H_{d}\left(F_{\mathcal{S}}^{[n]}(B), A_{\mathcal{S}}\right)=0
$$

for each $B \in P_{b, c l}(X)$.

Justification of Claim 1. Let us consider for arbitrarily chosen, but fixed, $B \in P_{b, c l}(X)$ and $b \in B$, the function $g \in \hat{\mathcal{C}}(X)$ given by $g(\omega, x)=\left\{\begin{array}{ll}x, & x \in B \\ b, & x \notin B\end{array}\right.$ for every $(\omega, x) \in \Lambda(I) \times X$.

Then

$$
\lim _{n \rightarrow \infty} d_{u}\left(H_{\mathcal{S}}^{[n]}(g), p\right)=0
$$

We have

$$
F_{\mathcal{S}}^{[n]}(M)=\overline{\bigcup_{\omega \in \Lambda(I)} f_{[\omega]_{n}}(M)}
$$

for every $n \in \mathbb{N}^{*}$ and every $M \in P_{b, c l}(X)$.

Indeed, relation (3.3) is valid for $n=1$ just by the definition of $F_{\mathcal{S}}$.

Let us suppose that it is true for $n$.

Then

$$
\begin{aligned}
& F_{\mathcal{S}}^{[n+1]}(M)=F_{\mathcal{S}}^{[n]}\left(F_{\mathcal{S}}(M)\right)=\overline{\bigcup_{\omega \in \Lambda(I)} f_{[\omega]_{n}}\left(F_{\mathcal{S}}(M)\right)}= \\
& =\overline{\bigcup_{\omega \in \Lambda(I)} f_{[\omega]_{n}}\left(\overline{\bigcup_{i \in I} f_{i}(M)}\right)} \stackrel{f_{[\omega]_{n}} \text { continuous }}{\subseteq} \overline{\left.\bigcup_{\omega \in \Lambda(I)} \overline{f_{[\omega]_{n}}\left(\cup_{i \in I} f_{i}(M)\right.}\right)}= \\
& =\overline{\bigcup_{\omega \in \Lambda(I)} f_{[\omega]_{n+1}}(M)}=\overline{\bigcup_{i \in I} f_{i}\left(\bigcup_{\omega \in \Lambda(I)} f_{[\omega]_{n}}(M)\right)} \subseteq \\
& \subseteq \overline{\bigcup_{i \in I} f_{i}\left(\overline{\bigcup_{\omega \in \Lambda(I)} f_{[\omega]_{n}}(M)}\right)}=\overline{\bigcup_{i \in I} f_{i}\left(F_{\mathcal{S}}^{[n]}(M)\right)}= \\
& =F_{\mathcal{S}}\left(F_{\mathcal{S}}^{[n]}(M)\right)=F_{\mathcal{S}}^{[n+1]}(M) .
\end{aligned}
$$


Hence, by mathematical induction method, we proved (3.3) .

We also have

$$
H_{\mathcal{S}}^{[n]}(g)(\Lambda(I) \times X)=\bigcup_{\omega \in \Lambda(I)} f_{[\omega]_{n}}(B)
$$

for every $n \in \mathbb{N}^{*}$.

Indeed,

$$
\begin{aligned}
H_{\mathcal{S}}^{[n]}(g)(\Lambda(I) \times X) & =\underset{\omega \in \Lambda(I), x \in X}{\cup} H_{\mathcal{S}}^{[n]}(g)(\omega, x)= \\
& \stackrel{\operatorname{Remark} 3.5}{=} \underset{\omega \in \Lambda(I), x \in X}{=} f_{[\omega]_{n}}\left(g\left(\omega_{n+1} \omega_{n+2} \ldots \omega_{m} \ldots, x\right)\right)^{g(\Lambda(I) \times B)=B}= \\
& =\underset{\omega \in \Lambda(I)}{\cup} f_{[\omega]_{n}}(B)
\end{aligned}
$$

for every $n \in \mathbb{N}^{*}$.

From (3.3) and (3.4) we get

$$
\overline{H_{\mathcal{S}}^{[n]}(g)(\Lambda(I) \times X)}=F_{\mathcal{S}}^{[n]}(B)
$$

for every $n \in \mathbb{N}^{*}$.

Note that

$$
H_{d}\left(F_{\mathcal{S}}^{[n]}(B), A_{\mathcal{S}}\right) \leq d_{u}\left(H_{\mathcal{S}}^{[n]}(g), p\right)
$$

for every $n \in \mathbb{N}^{*}$.

Indeed, we have

$$
\begin{array}{r}
H_{d}\left(F_{\mathcal{S}}^{[n]}(B), A_{\mathcal{S}}\right) \stackrel{(3.5)}{=} \mathcal{H}_{d}\left(\overline{H_{\mathcal{S}}^{[n]}(g)(\Lambda(I) \times X)}, \overline{p(\Lambda(I) \times X)}\right) \\
\stackrel{\text { Remark 2.1, a) }}{=} \mathcal{H}_{d}\left(H_{\mathcal{S}}^{[n]}(g)(\Lambda(I) \times X), p(\Lambda(I) \times X)\right)= \\
=\mathcal{H}_{d}\left(\underset{\omega \in \Lambda(I), x \in X}{\cup} H_{\mathcal{S}}^{[n]}(g)(\omega, x), \underset{\omega \in \Lambda(I), x \in X}{\cup} p(\omega, x)\right) \leq \\
\quad \underset{\operatorname{Remark} 2.1, \mathrm{~b})}{\leq} \sup _{\omega \in \Lambda(I), x \in X} \mathcal{H}_{d}\left(\left\{H_{\mathcal{S}}^{[n]}(g)(\omega, x)\right\},\{p(\omega, x)\}\right)= \\
=\sup _{\omega \in \Lambda(I), x \in X} d\left(H_{\mathcal{S}}^{[n]}(g)(\omega, x), p(\omega, x)\right)=d_{u}\left(H_{\mathcal{S}}^{[n]}(g), p\right)
\end{array}
$$

for every $n \in \mathbb{N}^{*}$.

In view of (3.2), by passing to limit as $n \rightarrow \infty$ in (3.6), we conclude that

$$
\lim _{n \rightarrow \infty} H_{d}\left(F_{\mathcal{S}}^{[n]}(B), A_{\mathcal{S}}\right)=0 .
$$

Claim 2.

$$
F_{\mathcal{S}}\left(A_{\mathcal{S}}\right)=A_{\mathcal{S}}
$$

Justification of Claim 2. For a fixed $K \in P_{c p}(X)$ we have

$$
\begin{gathered}
F_{\mathcal{S}}\left(A_{\mathcal{S}}\right) \stackrel{\text { Claim } 1}{=} F_{\mathcal{S}}\left(\lim _{n \rightarrow \infty} F_{\mathcal{S}}^{[n]}(K)\right) \stackrel{\text { Remark 2.2, a) }}{=} \lim _{n \rightarrow \infty} F_{\mathcal{S}}\left(F_{\mathcal{S}}^{[n]}(K)\right)= \\
=\lim _{n \rightarrow \infty} F_{\mathcal{S}}^{[n+1]}(K) \stackrel{\text { Claim } 1}{=} A_{\mathcal{S}} .
\end{gathered}
$$

iv)

a) As for a fixed $x_{0} \in X$ we have $\pi(\omega)=p\left(\omega, x_{0}\right)$ for every $\omega \in \Lambda(I)$ we infer that $\pi$ is continuous as $p$ is continuous.

Moreover, since $A_{\mathcal{S}}=p\left(\Lambda(I) \times\left\{x_{0}\right\}\right)=\pi(\Lambda(I))$ we conclude that $\pi$ is onto. 
b) We have

$$
\left(\pi \circ \tau_{i}\right)(\omega)=\pi(i \omega)=p\left(i \omega, x_{0}\right)=H_{\mathcal{S}}(p)\left(i \omega, x_{0}\right)=f_{i}\left(p\left(\omega, x_{0}\right)\right)=f_{i}(\pi(\omega))=\left(f_{i} \circ \pi\right)(\omega),
$$

for every $\omega \in \Lambda(I)$.

Now we are able to state our main result.

Theorem 3.4. For an iterated function system $\mathcal{S}=\left((X, d),\left(f_{i}\right)_{i \in I}\right)$, the following statements are equivalent:

1. There exists a comparison function $\varphi$ such that $\mathcal{S}$ is hyperbolic $\varphi$-contractive.

2. $\mathcal{S}$ is hyperbolic locally uniformly point fibred.

3. $\mathcal{S}$ is hyperbolic uniformly point fibred.

4. $\mathcal{S}$ is a hyperbolic diameter diminishing to zero iterated function system.

5. $\mathcal{S}$ has hyperbolic attractor and there exists a continuous surjection $\pi: \Lambda(I) \rightarrow A_{\mathcal{S}_{1}}$ such that $\pi \circ \tau_{i}=f_{i} \circ \pi$ for all $i \in I$, where $\mathcal{S}_{1}$ is the IFS mentioned in Definition 2.11, iii).

6. There exists a distance $d_{1}$ on $X$ such that

i) $d$ and $d_{1}$ are topologically equivalent;

ii) $\left(X, d_{1}\right)$ is complete;

iii) $H_{\mathcal{S}_{1}}$ is a Picard operator, where $\mathcal{S}_{1}=\left(\left(X, d_{1}\right),\left(f_{i}\right)_{i \in I}\right)$.

Proof. In view of Theorem 2.2 the statements 1), 2), 3), 4) and 5) are equivalent.

Proposition 3.2 ensures us that the implication 1$) \Rightarrow 6$ ) is valid.

Theorem 3.3 guarantees the validity of the implication 6$) \Rightarrow 5$ ).

A closer look at the case of iterated function systems comprising just one element yields the following:

Corollary 3.1. For a complete metric space $(X, d)$ and $f: X \rightarrow X$ a continuous function such that $f(B) \in P_{b}(X)$ for each $B \in P_{b}(X)$, the following properties are equivalent:

1. There exist a comparison function $\varphi$ and a distance $d_{1}$ on $X$ such that:

i) $d$ and $d_{1}$ are topologically equivalent;

ii) $\left(X, d_{1}\right)$ is complete;

iii) $f$ is $\varphi$-contractive with respect to $d_{1}$.

2. There exist $x^{*} \in X$ and a distance $d_{1}$ on $X$ such that:

i) $d$ and $d_{1}$ are topologically equivalent;

ii) $\left(X, d_{1}\right)$ is complete;

iii) $\lim _{n \rightarrow \infty} \sup _{x \in B} d_{1}\left(f^{[n]}(x), x^{*}\right)=0$ for every $B \in P_{b, c l}(X)$.

3. There exists a distance $d_{1}$ on $X$ such that:

i) $d$ and $d_{1}$ are topologically equivalent;

ii) $\left(X, d_{1}\right)$ is complete;

iii) for every $B \in P_{b, c l}(X)$ there exists $M_{B} \in P_{b, c l}(X)$ with the following properties: $B \subseteq$ $M_{B}, f\left(M_{B}\right) \subseteq M_{B}$ and $\lim _{n \rightarrow \infty} \sup _{x, y \in M_{B}} d_{1}\left(f^{[n]}(x), f^{[n]}(y)\right)=0$.

4. There exists a distance $d_{1}$ on $X$ such that:

i) $d$ and $d_{1}$ are topologically equivalent;

ii) $\left(X, d_{1}\right)$ is complete;

iii) there exists a $d_{1}$ bounded function $p:\{\omega\} \times X \rightarrow X$ with the following properties: $f \circ p=p$ and $\lim _{n \rightarrow \infty} \sup _{x \in X} d_{1}\left(\left(f^{[n]} \circ g\right)(\omega, x), p(\omega, x)\right)=0$ for every $d_{1}$ bounded function $g:\{\omega\} \times X \rightarrow X$, where $\omega$ is the unique element of the shift space associated to a singleton.

The last part of our paper is devoted to some fixed point flavored comments generated by the above corollary. 
Remark 3.6. Statement 2 of Corollary 3.1 implies that $f$ is a Picard operator, with respect to $d_{1}$, whose fixed point is $x^{*}$, so Statement 3 of the same Corollary could be seen as a sufficient condition for the existence and uniqueness of a fixed point of $f$.

Remark 3.7. The implications $\mathbf{3} \Rightarrow \mathbf{1}$ and $\mathbf{2} \Rightarrow \mathbf{1}$ from Corollary 3.1 are kind of converses to Browder's fixed point theorem (see Theorem 2.1) in the spirit of the Janos' and Leader's results (see [9] and [11]) which are converses to Banach's fixed point theorem.

Remark 3.8. Note that the image of the function $p$ whose existence is stated in Statement 4 of Corollary 3.1 has just one element (see Theorem 3.3, i)), let us say $x^{*}$, which is the fixed point of $f$ and that $\lim _{n \rightarrow \infty} d_{1}\left(f^{[n]}(x), x^{*}\right)=0$ for every $x \in X$, so $f$ is a Picard operator with respect to $d_{1}$.

Acknowledgment. We want to thank the referees whose generous and valuable remarks and comments brought improvements to the paper and enhanced clarity.

\section{REFERENCES}

[1] Atkins, R., Barnsley, M., Vince, A. and Wilson, D., A characterization of hyperbolic affine iterated function systems, Topology Proc., 36 (2010), 189-211

[2] Banakh, T., Kubiś, W., Novosad, N., Nowak, M. and Strobin, F., Contractive function systems, their attractor and metrization, Topol. Methods Nonlinear Anal., 46 (2015), 1029-1066

[3] Barnsley, M., Fractals Everywhere, Academic Press, Boston, MA, 1988

[4] Barnsley, M. and Vince, A., Real projective iterated function systems, J. Geom. Anal., 22 (2012), 1137-1172

[5] Barnsley M., Leśniak K., On the continuity of the Hutchinson operator, Symmetry, 7 (2015), 1831-1840

[6] Browder, F., On the convergence of successive approximations for nonlinear functional equations, Indag. Math., 30 (1968), 27-35

[7] Hutchinson, J., Fractals and self similarity, Indiana Univ. Math. J., 30 (1981), 713-747

[8] Jachymski, J., Around Browder's fixed point theorem for contractions, J. Fixed Point Theory Appl., 5 (2009), $47-61$

[9] Janos, L., A converse of Banach's contraction theorem, Proc. Amer. Math. Soc., 18 (1967), 287-289

[10] Kameyama, A., Distances on topological self-similar sets and the kneading determinants, J. Math. Kyoto Univ., 40 (2000), 603-674

[11] Leader, S., A topological characterization of Banach contractions, Pacific J. Math., 69 (1977), 461-466

[12] Miculescu, R. and Mihail, A., Alternative characterization of hyperbolic infinite iterated function systems, J. Math. Anal. Appl., 407 (2013), 56-68

[13] Miculescu, R. and Mihail, A., On a question of A. Kameyama concernin self-similar metrics, J. Math. Anal. Appl., 422 (2015), 265-271

[14] Miculescu, R. and Mihail, A., A sufficient condition for a finite family of continuous functions to be transformed into $\psi$-contractions, Ann. Acad. Sci. Fenn., Math., 41 (2016), 51-65

[15] Miculescu, R. and Mihail, A., Remetrization results for possibly infinite self-similar systems, Topol. Methods Nonlinear Anal., 47 (2016), 333-345

[16] Miculescu, R. and Mihail, A., A generalization for a finite family of functions of the converse of Browder's fixed point theorem, Bull. Braz. Math. Soc. (N.S.), 49 (2018), 673-698

[17] Miculescu, R. and Mihail, A., Diameter diminishing to zero IFSs, arXiv:2101.12705

[18] Mihail, A., The canonical projection between the shift space of an IIFS and its attractor as a fixed point, Fixed Point Theory Appl., 15 (2015), No. 75

[19] Munkres, J., Topology, 2nd edn. Prentice Hall Inc., Englewood Cliffs, NJ (2000)

[20] Urziceanu, S., Alternative characterizations of AGIFSs having attractor, Fixed Point Theory, 20 (2019), 729-740

[21] Vince, A., Mobius iterated function systems, Trans. Amer. Math. Soc., 365 (2013), 491-509

${ }^{1,3}$ TRANSILVANIA UNIVERSITY OF BRAŞOV

FACULTY OF MATHEMATICS AND COMPUTER SCIENCE

IULIU MANIU 50, 500091, BRAŞOV, ROMANIA

Email address: radu.miculescu@unitbv.ro

Email address: cristina.pacurar@unitbv.ro

${ }^{2}$ UNIVERSITY OF BUCHAREST

FACULTY OF MATHEMATICS AND COMPUTER SCIENCE

ACADEMIEI 14, 010014, BUCHAREST, ROMANIA

Email address: mi hail_alex@yahoo.com 